ISSN-PRINT 1794-9831 / E-ISSN 2322-7028

Vol. $19 \mathrm{~N}^{\mathrm{o}} 1$ / ene - abr, 2022 / Cúcuta, Colombia.

do https://doi.org/10.22463/17949831.3114

Original

\title{
Evaluación de la hoja de enfermería de una Unidad de Cuidados Intensivos
}

\author{
Evaluation of the nursing sheet of an Intensive Care Unit
}

Avaliação das anotações de enfermagem de uma Unidade de Terapia Intensiva

\author{
Aracely Díaz-Oviedo ${ }^{1}$ \\ José Martín Castro-Mata ${ }^{2}$
}

\section{Resumen}

Objetivo: Evaluar el nivel del cumplimiento de la hoja de registros clínicos de enfermería de una Unidad de Cuidados Intensivos en México. Materiales y Métodos: Se realizó un estudio cuantitativo, descriptivo transversal, en una Unidad de Cuidados Intensivos de un hospital en San Luis Potosí, México, en diciembre 2020-enero 2021. Se utilizó una muestra de 150 hojas de registro clínicos de enfermería, evaluando los 40 estándares básicos del registro, con el instrumento "Cedula para evaluar la eficacia de la práctica de enfermería" de la Comisión Nacional de Arbitraje Médico en México; seguidamente los datos se analizaron con estadística descriptiva en el programa SPSS, versión 18. Se siguieron las recomendaciones de la Declaración de Helsinki y los principios éticos de confidencialidad que establece la institución. Resultados: Se identifica un nivel de cumplimiento deficiente de estándares, donde: 2 estándares cumplen parcialmente, 23 totalmente, 11 no cumplen con lo que establece la Comisión Nacional de Arbitraje Médico, y 4 que el formato no contiene, se anexan al mismo por el personal de enfermería, a través de estadística descriptiva. Igualmente, se realizó un análisis estadístico, donde se obtuvo como moda y mediana del cumplimiento general el $100 \%$, y una media de cumplimiento de $67.26 \%$. Conclusiones: Al encontrar un nivel deficiente de cumplimiento de estándares se construye una propuesta de nuevo formato para la institución de salud, que logre dar cumplimiento total con los elementos establecidos en la Norma Oficial Mexicana 004-SSA3-2012, del Expediente Clínico y la Comisión Nacional de Arbitraje Médico.

Palabras clave: Nivel de cumplimiento; registros; atención de enfermería; cuidado critico.

\section{Abstract}

Objective: To evaluate the level of compliance with the nursing clinical record sheet of an Intensive Care Unit in Mexico. Materials and methods: A quantitative, descriptive cross-sectional study was carried out in an Intensive Care Unit of a hospital in San Luis Potosí, Mexico, in December 2020-January 2021, with a sample of 150 nursing clinical record sheets, the 40 basic standards of the registry, with the instrument "Cedula to evaluate the effectiveness of nursing practice" of the National Commission of Medical Arbitration in Mexico, the data were analyzed with descriptive statistics in the SPSS program. Version 18, and the recommendations of the Declaration of Helsinki and ethical principles of confidentiality established by the institution were followed. Results: A deficient level of compliance with standards is identified, where 2 standards partially comply, 23 fully, 11 do not comply with what, es-

\section{Autor de correspondencia"}

1* Profesor Investigador de Tiempo Completo, SNI I (Secretaría de Investigación de la Facultad de Enfermería y Nutrición de la Universidad Autónoma de San Luis Potosí. San Luis Potosí, México. Correo: aracelydiaz@uaslp. $\underline{\mathrm{mx}}$ (D) 0000-0001-9168-9859

2 Enfermero Especialista en Cuidado Critico del Hospital General del ISSSTE. Delegación San Luis Potosí Potosí. San Luis Potosí, México. Correo: nitram465@hotmail.com (iD 0000-0001-7166-9041

Recibido: 13 agosto 2021

Aprobado: 28 octubre 2021

\section{Para citar este artículo}

Diaz-Oviedo A, Castro-Mata JM. Evaluación de la hoja de enfermería de una Unidad de Cuidados Intensivos. Rev. cienc. cuidad. 2022; 19(1):19-30. https://doi. org/10.22463/17949831.3114

(C) Universidad Francisco de Paula Santander. Este es un artículo bajo la licencia CC-BY-NC-ND 


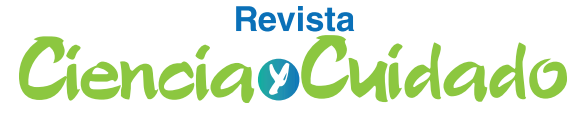

Aracely Díaz-Oviedo, José Martín Castro-Mata

\section{Scientific Journal of Nursing}

tablished by the National Medical Arbitration Commission, and 4 that the format does not contain, are attached to it A statistical analysis was performed by the nursing staff through descriptive statistics, where the mode and median of general compliance was $100 \%$, and a mean compliance of $67.26 \%$. Conclusions: Upon finding a deficient level of compliance with standards, a proposal for a new format is built for the health institution, which achieves full compliance with the elements established in the Official Mexican Standard 004-SSA3-2012, of the Clinical Record and the National Commission of Medical Arbitration.

Keywords: Compliance level, Records; Nursing care; Critical Care.

\section{Resumo}

Objetivo: Avaliar o nível de cumprimento das anotações de enfermagem nos prontuários de pacientes de uma Unidade de Terapia Intensiva no México. Materiais e métodos: Realizou-se um estudo quantitativo, descritivo, transversal, numa Unidade de Terapia Intensiva num hospital em San Luis Potosí, Mexico, entre dezembro de 2020 e janeiro de 2021. Usou-se uma amostra de 150 folhas de anotações clínicas de enfermagem, avaliando os 40 padrões básicos de registro, com o instrumento "Cartão para avaliar a eficácia da prática de enfermagem-CERCE" da Comissão Nacional de Arbitragem Médica em México; posteriormente os dados foram analisados usando o programa SPSS, versão 18. Seguiram-se as recomendações da Declaração de Helsinki e os princípios de confidencialidade estabelecidos pelo hospital. Resultados: Identificou-se um nível de cumprimento deficiente de padrões, onde: 2 padrões cumprem parcialmente, 23 totalmente e 11 não cumprem os requerimentos estabelecidos pela Comissão Nacional de Arbitragem Médica, 4 que o instrumento não contem, são anexados pelo pessoal de enfermagem usando estatística descritiva. Igualmente a analise estatística mostrou que a moda e mediana do cumprimento geral foi do $100 \%$ e uma media de 67,26\%. Conclusão: Ao identificar a deficiência no cumprimento de padrões se constrói a proposta de um novo formato para o hospital, que consiga cumprir totalmente os critérios estabelecidos na Norma Oficial Mexicana 004-SSA3-2012, do prontuário clínico e a Comissão de Arbitragem Médica.

Palavras-chave: Fidelidade a diretrizes; registros; cuidados de enfermagem; cuidados críticos.

\section{Introducción}

Los registros clínicos de enfermería datan del siglo XIX, con Florence Nightingale, máxima representante de la enfermería a nivel mundial, quien al observar las condiciones tan deplorables y poco higiénicas en las que eran atendidos los soldados durante la Guerra de Crimea (1), señalaba en sus notas de enfermería, que el personal de enfermería es el encargado de cuidar y ayudar al paciente que sufre alguna enfermedad a vivir y mantener la salud, atenciones que deben ir desde un niño o una persona sana, con la finalidad de protegerlos ante la susceptibilidad de padecer alguna enfermedad (2). Lo planteado anteriormente evidencia la importan- cia de la nota de enfermería, en donde se indiquen las condiciones en las que se encuentran los pacientes.

Debe señalarse que los formatos y registros de enfermería garantizan un seguimiento permanente del tratamiento del paciente y la protección legal de la enfermera; de igual forma permiten identificar al personal que estuvo a cargo de un paciente determinado, analizar la oportunidad de la atención y realizar la vigilancia de enfermería, involucrando además la ética profesional, revelando la evidencia de la historia y la cultura profesional (3-6). A pesar de que se tiene plenamente identificada su importancia, dado que responden a exigencias de carácter legal, institucional y compromiso 


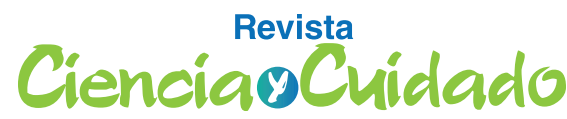

Scientific Journal of Nursing

profesional, la realidad muestra que sin embargo los profesionales de enfermería, incumplen con su registro, derivado del poco valor que se les otorga (7-8).

Por otra parte, las guías científicas sugieren que los registros de enfermería deben ser cuidadosamente evaluados por la Comisión Conjunta Internacional (JCI) (9), con el propósito de evaluar la calidad de la atención y la seguridad del cuidado lo cual debe ser objetivo permanente de análisis. Por su parte en México, la Norma Oficial Mexicana 168-SSA1-1998, del Expediente Clínico, señala que se le considera como documento de cualquier registro médico realizado por el personal de salud, en donde se presentan los hechos y actos relativos a la atención prestada a una persona determinada. En este sentido, la hoja de enfermería constituye un elemento fundamental (10) que según la Norma Oficial Mexicana 004-SSA3-2012, del Expediente Clínico, señala que la hoja de registros clínicos de enfermería, debe contener cinco elementos como son: habitus exterior, gráfica de signos vitales, administración de medicamentos, procedimientos realizados y observaciones de enfermería (11).

Cabe destacar que la unidad de cuidados intensivos (UCI) es un área de salud especializada, donde se brinda atención a pacientes inestables que requieren vigilancia y monitorización continua. Estos cuidados de enfermería comenzaron a desarrollarse en la década de los 60 en Estados Unidos, con un fuerte desarrollo en Inglaterra, Australia y Canadá, y posteriormente en Latinoamérica (12-13). El contar con una sofisticación tecnológica y el tener evidencia del registro enfermero, han impactado positivamente en la evolución del paciente crítico (14). En México, los hospitales ofrecen dos opciones de cuidado: las unidades de hospitalización convencional y las UCIS, en donde la enfermera(o) intensivista, representa el mayor usuario de la tecnología e información sanitaria (15) quien al realizar registros de enfermería completos, asegurara la circulación de información entre los diferentes actores involucrados, mejorando la calidad y seguridad de la atención (16-17).

Se puede indicar que la legislación de los registros clínicos de enfermería perfecciona un marco regulatorio, mejora de la calidad en la atención y genera un cuidado libre de riesgos (18). En México actualmente dentro del marco legal de los registros clínicos de enfermería, se encuentra la Norma Oficial Mexicana 004-
Evaluación de la hoja de enfermería de una Unidad

de Cuidados Intensivos
SSA3-2012, del Expediente Clínico (11), en donde se establecen los 40 estándares básicos de la Comisión Nacional de Arbitraje Médico referidos en la "Cedula para evaluar la eficacia de la práctica de Enfermería”. Esta Norma fue creada en el año 2017, con la finalidad de responder de manera integral al desempeño del profesional de enfermería (19).

De modo similar se puede decir que los expedientes clínicos son documentos que se elaboran a todo paciente al ingresar a una institución de salud, los cuales deben contener los datos generales y específicos de la enfermedad tales como: diagnóstico del paciente, historia clínica y observaciones entre otros. Por tanto, este es un documento médico-legal, y uno de los más importantes que contiene el expediente clínico, debido a que es básico por su contenido. En este contexto, es la enfermera(o) la persona que tiene mayor capacidad para dar a conocer la evolución del paciente, a través de sus registros, función que realiza como mínimo tres veces diarias (20). Es decir, que en el expediente médico se deben registrar las necesidades del paciente, las conductas clínicas de atención que fueron implementadas y la evaluación continua de la atención brindada (21).

De ahí, que los formatos y registros de enfermería sean actualmente de suma importancia, debido al aumento de las situaciones médico-legales, frente a las cuales es indispensable contar con un respaldo del trabajo realizado que idealmente, debe ser: objetivo, preciso, claro, legible y simultaneo (22). En el año 2001, las enfermeras formaron un grupo de calidad orientado a evaluar cómo se estaban realizando los registros de enfermería en la Clínica Alemana y en 2002 se integraron a este grupo las auxiliares de enfermería, con lo que se lograron mejores resultados (23).

Por otro lado, se requiere que sean legibles y de fácil acceso ya que, son piezas fundamentales en el cuidado otorgado al paciente, puesto que apoyan, mantienen y favorecen la calidad de la atención clínica; además representan una fuente de información del estado de salud del paciente. Hay que indicar que pueden ser importantes para la evaluación en la toma de decisiones y también se les considera como documentos legales que fundamentan los actos del equipo de salud. Igualmente, deben permitir que se les analice con fines de investigación en tanto que, el registro en el formato permite identificar al personal que estuvo a cargo de un deter- 


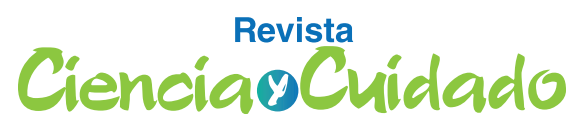

Scientific Journal of Nursing

minado paciente, analizar la forma de la atención y realizar la vigilancia de enfermería (24).

Las características y calidad de los formatos de registros clínicos de enfermería en el área de UCI, han sido objeto de investigación, puesto que son definidos como la forma de comunicación escrita que debe ser clara y concisa, lo que ha posibilitado una mejor interacción entre los miembros del equipo de salud (25), ya que reflejan de manera significativa el proceso de continuidad sobre la atención que proporciona el profesional de enfermería a los pacientes.

Cabe señalar que los elementos que conforman la hoja de registros clínicos de enfermería en algunas Unidades de Cuidados Intensivos son diversos respecto a los criterios que establece la CONAMED ya que, en estas unidades se ha podido identificar que el formato carece, en algunos casos, de estos criterios.

Es necesario recalcar que la documentación de la práctica de enfermería es indispensable que sea correcta en formato e información, de tal forma que sirva para evaluar, mejorar la eficacia y la productividad de los profesionales de salud; al respecto el eje central de este estudio es evaluar el nivel de cumplimiento de la hoja de enfermería de una Unidad de Cuidados Intensivos en una institución pública.

\section{Objetivos}

Evaluar el nivel de cumplimiento de la hoja de enfermería de Unidad de Cuidados Intensivos.

\section{Objetivos Específicos}

- Identificar el nivel de cumplimiento de los estándares en los registros clínicos de enfermería en la UCI.

- Identificar las medidas de tendencia central del cumplimiento de los estándares en general, sobre el formato de la hoja de registros clínicos de enfermería referidos por la Comisión Nacional de Arbitraje Médico (CONAMED).

\section{Materiales y Métodos}

Se realizó un estudio cuantitativo y descriptivo transversal centrado en el nivel de cumplimiento de la hoja de enfermería de la Unidad de Cuidados Intensivos (UCI).

La población de estudio estuvo conformada por formatos de registros clínicos de enfermería, de una UCI en San Luis Potosí, México, durante el periodo comprendido entre diciembre de 2020 y enero de 2021. Para ello se realizó una revisión del cumplimiento de cada uno de los estándares, con una duración de 10 minutos para cada hoja de enfermería.

A continuación, se seleccionaron las hojas de registros de enfermería pertenecientes a la UCI, que se encontraron en el área de archivo de la institución; al mismo tiempo se excluyeron las hojas de enfermería de servicios institucionales que no pertenecieron a la UCI y aquellos formatos de hojas de pacientes con diagnóstico de SARS-Cov2, por seguridad del investigador.

Finalmente, la muestra la conformaron 150 hojas de registros clínicos de enfermería. Esta muestra se efectuó por conveniencia en función del acceso a la institución por la pandemia de COVID-19; por tanto su tamaño se calculó conforme al universo del formatos de las hojas de enfermería (26).

El nivel de cumplimiento se midió de acuerdo a la cédula para medir la eficacia de la práctica de enfermería, referida por la CONAMED, conformada por 40 estándares que evalúan en conjunto el cumplimiento de los formatos de hojas de enfermería. Desde este punto de vista se adaptaron niveles de cumplimiento, en donde los 40 estándares presentan 2 opciones de respuesta: cumplimiento al $100 \%$ y no cumplimiento; sin embargo, se pudieron identificar 2 opciones más, que mostraban el cumplimiento parcial y los estándares anexados por el personal de enfermería a la de hoja. Por tanto el nivel de cumplimiento con mayor apego a los criterios de la CONAMED, se evalúa como excelente, mientras el de menor cumplimiento como deficiente.

De igual forma, se utilizó la "Cedula para evaluar la eficacia de la práctica de enfermería", diseñada y creada por la CONAMED, conformada por 40 estándares que evalúan en conjunto el cumplimiento de los formatos de hojas de enfermería, los cuales presentan 2 opciones de respuesta: $1=$ cumplimiento al $100 \%$ y $2=$ sin cumplimiento.

Teniendo en cuenta lo expuesto anteriormente, la evaluación del nivel de cumplimiento de la hoja de enfer- 


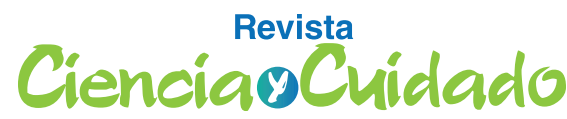

Scientific Journal of Nursing

mería, se basó e la "Cédula para evaluar la eficacia de la práctica de la enfermería" a fin de determinar la eficacia de la práctica de enfermería referida por la $\mathrm{CON}$ AMED. Sin embargo, se añadieron 2 opciones más de respuesta no incluidas en la cédula, con la finalidad de completar los estándares: 3=estándares con cumplimiento parcial y $4=$ estándares anexados. Por tanto, al no existir una escala para su evaluación se realizó la siguiente: evaluación Excelente: cumplimiento del $100-91 \%$ de los estándares en conjunto; evaluación Muy Buena: cumplimiento del 90-81\% de los estándares en conjunto; evaluación Buena: cumplimiento del $80-71 \%$ de los estándares en conjunto; evaluación Regular: cumplimiento del 70-61\% de los estándares en conjunto y evaluación Deficiente: cumplimiento menor del $60 \%$ de los estándares en conjunto.

Los datos se analizaron a través del programa estadístico SPSS Versión 18, mediante estadística inferencial con tablas de frecuencias y porcentajes, en relación al nivel de cumplimiento general de los 40 estándares básicos de la CONAMED, así como también del nivel de adaptación de cumplimiento.

Hay que mencionar además que la investigación fue aprobada por el Comité de Ética e Investigación de la Facultad de Enfermería y Nutrición (CEIFE), de la Universidad Autónoma de San Luis Potosí, (CEIFE-2020-329). De manera semejante se consiguió la aprobación del Comité de ética e investigación del Hospital de seguridad social donde se realizó el estudio en San Luis Potosí con registro interno:007/2020. Por todo lo anterior se demuestra que se respetó lo establecido por el código de declaración de Helsinki establecido por la $18^{\text {a }}$ Asamblea Médica Mundial, Helsinki, Finlandia, junio 1964, y enmendada por la $29^{\text {a }}$ Asamblea Medica Mundial, respetando la importancia de la validez de la muestra de estudio. Así mismo, se aclara que la investigación se realizó con fines educativos y bajo el conocimiento y revisión de bibliografía científica, por tanto los resultados obtenidos se publicaron respetando lo establecido en la declaración de Helsinki (27). Igualmente, se acataron las disposiciones del Título Segundo de la Ley General de Salud en Materia de Investigación, en donde se establece que toda investigación contará con el dictamen favorable de los Comités de Investigación, de Ética en Investigación y de Bioseguridad (Capítulo I, Art. 14 Fracc. VII). A partir de las características y consideraciones estipuladas en el reglamento, el estudio fue considera-
Evaluación de la hoja de enfermería de una Unidad

de Cuidados Intensivos do como una investigación sin riesgo, en virtud de que se emplearon técnicas y métodos de investigación documental, a través de la revisión de expedientes clínicos y otros, (Titulo II Capítulo I Art. 17, Fracc. I) (28). Es por esta razón que se tuvo en cuenta la carta de no conflicto de intereses, la cual señala que no deberán existir ningún tipo de problema antes, durante y después de la investigación (29).

En este sentido se acataron los puntos del Decálogo del Código de Ética para las Enfermeras y Enfermeros de México, tomando como principio básico en la investigación la confidencialidad y privacidad de la información que contenían los formatos de hojas de enfermería. Es por eso, que se retuvieron los instrumentos durante la recopilación de los datos y se desecharon cuando ya se transcribieron digitalmente al paquete de datos estadísticos. Además, se puso en práctica lo establecido en el capítulo V, de los deberes de las enfermeras para con su profesión, Art.24 el cual menciona la contribución al desarrollo de la profesión a través de la investigación (30). Del mismo modo, se envió una carta solicitando la autorización a la institución para hacer uso de la información que se utilizaría en la investigación. Por último, para la fundamentación del estudio se tomó en cuenta la Norma Oficial Mexicana NOM-004-SSA3-2012 del Expediente Clínico, la cual en su apartado 9.1 menciona que la hoja de registros clínicos de enfermería debe contener al menos con 5 elementos importantes tales como: habitus exterior, gráfica de signos vitales, administración de medicamentos con la fecha, hora, cantidad y vía, procedimientos realizados y observaciones de enfermería; de donde se deriva posteriormente, la cédula para evaluar la eficacia de la práctica de la enfermería relacionada con los 40 estándares básicos de la Comisión Nacional de Arbitraje Médico (CONAMED) (19).

\section{Resultados}

Se puede identificar en la Tabla 1, los porcentajes de cumplimiento de cada uno de los 40 estándares básicos establecidos por la Comisión Nacional de Arbitraje Médico; también, se observa que el formato de hoja de registros clínicos de enfermería de la Unidad de Cuidados Intensivos, de un hospital de San Luis Potosí, México, contiene únicamente 23 estándares que cumplen al $100 \%$ con los requerimientos de la CONAMED, los cuales representan un $57.5 \%$, por tanto se evalúa el nivel de cumplimiento del formato como deficiente. 


\section{Scientific Journal of Nursing}

Tabla 1. "Cumplimiento general de los estándares del formato de registros clínicos de enfermería de la Unidad de Cuidados Intensivos, de un hospital de San Luis Potosí, México, de acuerdo a lo establecido por la Comisión Nacional de Arbitraje Médico"

\begin{tabular}{|c|c|c|c|}
\hline Estándar & Nombre del Estándar & f & $\%$ \\
\hline 1 & Identificación & 150 & 75 \\
\hline 2 & Diagnóstico médico & 150 & 100 \\
\hline 3 & Frecuencia cardiaca & 150 & 100 \\
\hline 4 & Presión Arterial & 150 & 100 \\
\hline 5 & Presión Venosa Central & 150 & 0 \\
\hline 6 & Frecuencia Respiratoria & 150 & 100 \\
\hline 7 & Alteraciones en las constantes vitales & 150 & 100 \\
\hline 8 & Estatura & 150 & 0 \\
\hline 9 & Peso & 150 & 0 \\
\hline 10 & Perímetros & 150 & 0 \\
\hline 11 & Formula/Dieta/Líquidos Orales & 150 & 100 \\
\hline 12 & Ingesta del paciente & 150 & 100 \\
\hline 13 & Ayuno & 150 & 0 \\
\hline 14 & Líquidos Parenterales & 150 & 100 \\
\hline 15 & Elementos Sanguíneos & 150 & 100 \\
\hline 16 & Oportunidad en la administración de elementos sanguíneos & 150 & 100 \\
\hline 17 & Total de ingresos parenterales & 150 & 100 \\
\hline 18 & Control de líquidos & 150 & 100 \\
\hline 19 & Egresos: Uresis & 150 & 100 \\
\hline 20 & Egresos: Evacuaciones & 150 & 100 \\
\hline 21 & Egresos: Sangrado y succiones & 150 & 100 \\
\hline 22 & Total de Ingresos & 150 & 100 \\
\hline 23 & Total de Egresos & 150 & 100 \\
\hline 24 & Toma y solicitud de laboratorios & 39 & 26.0 \\
\hline 25 & Toma de reactivos: destrostix/glucocetonurias & 150 & 50 \\
\hline 26 & Programación de intervención quirúrgica & 150 & 0 \\
\hline 27 & Administración de medicamentos & 150 & 100 \\
\hline 28 & Realización de tratamientos & 150 & 100 \\
\hline 29 & Signos y síntomas & 150 & 100 \\
\hline 30 & Cuidados de enfermería & 150 & 100 \\
\hline 31 & Identificación de necesidades & 150 & 100 \\
\hline 32 & Intervenciones de enfermería & 150 & 100 \\
\hline 33 & Respuesta y evolución al tratamiento & 61 & 40.7 \\
\hline 34 & Responsable de brindar la atención & 111 & 74.0 \\
\hline 35 & Responsable de supervisar la atención & 150 & 0 \\
\hline 36 & Personal suficiente de acuerdo a los indicadores de calidad & 150 & 0 \\
\hline 37 & Perfiles profesionales & 150 & 0 \\
\hline 38 & Material & 150 & 0 \\
\hline 39 & Resultados: Ausencia de quejas o demandas & 150 & 0 \\
\hline 40 & Resultados: Ausencia de eventos adversos & 150 & 0 \\
\hline
\end{tabular}

Fuente: Elaboración propia 


\section{CienciagoCuidado}

\section{Scientific Journal of Nursing}

Por otro lado, en la Tabla 2 se visualiza que 23 estándares se adaptan en su totalidad al formato de registros clínicos, con una frecuencia de 150, siendo los siguientes: Diagnóstico médico; Frecuencia cardiaca; Presión Arterial; Frecuencia Respiratoria; Alteraciones en las constantes vitales; Formula/Dieta/Líquidos Orales; Ingesta del paciente; Líquidos Parenterales; Elementos Sanguíneos; Oportunidad en la administración

Tabla 2. Cumplimiento de los estándares del formato de registros clínicos de enfermería de la Unidad de Cuidados Intensivos, de un hospital de San Luis Potosí, México, de acuerdo a lo establecido por la Comisión Nacional de Arbitraje Médico.

\begin{tabular}{|c|c|c|c|c|c|c|c|c|c|}
\hline \multirow[t]{3}{*}{ Estándar } & \multirow[t]{3}{*}{ Nombre del Estándar } & \multicolumn{8}{|c|}{ Cumplimiento de estándares } \\
\hline & & \multicolumn{2}{|c|}{$\begin{array}{l}\text { Cumplimiento } \\
\text { al } 100 \%\end{array}$} & \multicolumn{2}{|c|}{$\begin{array}{l}\text { No dan cum- } \\
\text { plimiento }\end{array}$} & \multicolumn{2}{|c|}{$\begin{array}{l}\text { Cumplen } \\
\text { parcialmente }\end{array}$} & \multicolumn{2}{|c|}{$\begin{array}{l}\text { Anexados por } \\
\text { el personal }\end{array}$} \\
\hline & & $f$ & $\%$ & $f$ & $\%$ & $f$ & $\%$ & $f$ & $\%$ \\
\hline 1 & Identificación & & & & & 150 & 100 & & \\
\hline 2 & Diagnóstico médico & 150 & 100 & & & & & & \\
\hline 3 & Frecuencia cardiaca & 150 & 100 & & & & & & \\
\hline 4 & Presión Arterial & 150 & 100 & & & & & & \\
\hline 5 & Presión Venosa Central & & & 150 & 100 & & & & \\
\hline 6 & Frecuencia Respiratoria & 150 & 100 & & & & & & \\
\hline 7 & Alteraciones en las constantes vitales & 150 & 100 & & & & & & \\
\hline 8 & Estatura & & & 150 & 100 & & & & \\
\hline 9 & Peso & & & & & & & 150 & 100 \\
\hline 10 & Perímetros & & & 150 & 100 & & & & \\
\hline 11 & Formula/Dieta/Líquidos Orales & 150 & 100 & & & & & & \\
\hline 12 & Ingesta del paciente & 150 & 100 & & & & & & \\
\hline 13 & Ayuno & & & 150 & 100 & & & & \\
\hline 14 & Líquidos Parenterales & 150 & 100 & & & & & & \\
\hline 15 & Elementos Sanguíneos & 150 & 100 & & & & & & \\
\hline 16 & $\begin{array}{l}\text { Oportunidad en la administración de ele- } \\
\text { mentos sanguíneos }\end{array}$ & 150 & 100 & & & & & & \\
\hline 17 & Total de ingresos parenterales & 150 & 100 & & & & & & \\
\hline 18 & Control de líquidos & 150 & 100 & & & & & & \\
\hline 19 & Egresos: Uresis & 150 & 100 & & & & & & \\
\hline 20 & Egresos: Evacuaciones & 150 & 100 & & & & & & \\
\hline 21 & Egresos: Sangrado y succiones & 150 & 100 & & & & & & \\
\hline 22 & Total de Ingresos & 150 & 100 & & & & & & \\
\hline 23 & Total de Egresos & 150 & 100 & & & & & & \\
\hline 24 & Toma y solicitud de laboratorios & & & & & & & 39 & 26.0 \\
\hline 25 & $\begin{array}{l}\text { Toma de reactivos: destrostix/glucocetonu- } \\
\text { rias }\end{array}$ & & & & & 150 & 100 & & \\
\hline 26 & Programación de intervención quirúrgica & & & 150 & 100 & & & & \\
\hline 27 & Administración de medicamentos & 150 & 100 & & & & & & \\
\hline 28 & Realización de tratamientos & 150 & 100 & & & & & & \\
\hline 29 & Signos y síntomas & 150 & 100 & & & & & & \\
\hline
\end{tabular}


Scientific Journal of Nursing

\begin{tabular}{|c|c|c|c|c|c|c|c|}
\hline 30 & Cuidados de enfermería & 150 & 100 & & & & \\
\hline 31 & Identificación de necesidades & 150 & 100 & & & & \\
\hline 32 & Intervenciones de enfermería & 150 & 100 & & & & \\
\hline 33 & Respuesta y evolución al tratamiento & & & & & 61 & 40.7 \\
\hline 34 & Responsable de brindar la atención & & & & & 111 & 74.0 \\
\hline 35 & Responsable de supervisar la atención & & & 150 & 100 & & \\
\hline 36 & $\begin{array}{l}\text { Personal suficiente de acuerdo a los indica- } \\
\text { dores de calidad }\end{array}$ & & & 150 & 100 & & \\
\hline 37 & Perfiles profesionales & & & 150 & 100 & & \\
\hline 38 & Material & & & 150 & 100 & & \\
\hline 39 & $\begin{array}{l}\text { Resultados: Ausencia de quejas o deman- } \\
\text { das }\end{array}$ & & & 150 & 100 & & \\
\hline 40 & Resultados: Ausencia de eventos adversos & & & 150 & 100 & & \\
\hline
\end{tabular}

Fuente: Elaboración propia

Además, se identificaron 11 estándares que no cumplen con lo establecido por la Comisión Nacional de Arbitraje Médico, los cuales son: Presión Venosa Central; Estatura; Perímetros; Ayuno; Programación de intervención quirúrgica; responsable de supervisar la atención; Perfiles profesionales; Material; Resultados: Ausencia de quejas o demandas; Resultados: Ausencia de eventos adversos, con una frecuencia de 150 hojas, representando el $100 \%$ de cada uno de los estándares.

Cabe señalar que se pueden observar 2 estándares con cumplimiento parcial: el estándar 01 denominado como Identificación, en el que se deben indicar los registros clínicos de enfermería, solamente presenta: el Nombre de la Unidad Médica, Nombre y apellidos del paciente, Servicio, Cama y Fecha; obviando el Numero de afiliación, la Edad y el Sexo. Respecto al estándar 25 en donde se debe señalar la Toma de reactivos: destrostix/ glucocetonurias, solamente cumple con el destrostix.

Y finalmente se pudo encontrar que 4 estándares con los que no cumple la hoja de registros clínicos de enfermería, fueron anexados por el personal de enfermería de la siguiente manera: el estándar peso, con una frecuencia de 150 formatos de hojas, que equivalen al 100\%; el estándar toma y solicitud de laboratorios, con una frecuencia de 39 formatos de hojas correspondientes al 26.0\%; el estándar respuesta y evolución al tratamiento con una frecuencia de 61 y un porcentaje de 40.7\%; por último el estándar del Responsable que brinda la atención, con una frecuencia de $111 \mathrm{y}$ un porcentaje de $74.0 \%$.

\section{Discusión}

El nivel de cumplimiento general de la hoja de registros clínicos de enfermería de la UCI de un hospital público en San Luis Potosí, es deficiente, ya que solamente da cumplimiento a 23 estándares básicos, lo que representa un $57.5 \%$. Al respecto, se puede mencionar lo que dice Villa Suarez M. en su artículo de investigación titulado "Registros de Enfermería como Evidencia del Cuidado" en donde afirma que la ausencia de formatos de registros de enfermería completos de cuidados que se brindan a pacientes, puede entenderse como una falta legal y ética; de ahí la importancia de contar con formatos de calidad, que le permitan al personal de enfermería asumir la tarea del cuidado de los pacientes de manera responsable (31). Por el contrario, López et al. (32), refieren en su investigación titulada "Construcción y validación de un registro clínico para la atención asistencial de enfermería", que en la evaluación que realizaron se observó un nivel de excelencia, con un porcentaje total del $95.59 \%$ de cumplimiento de la hoja. De donde se concluye que es de vital importancia contar en las UCIS con formatos de registros clínicos de enfermería, que permitan al profesional de la enfermería plasmar en su totalidad el cuidado brindado.

Se debe precisar que el formato de hoja de registros clínicos de enfermería de la UCI, cuenta con dos estándares de cumplimiento parcial, como lo son el de: Identificación, en donde se ubica el Nombre de la Unidad Médica, Nombre y apellidos del paciente, Servicio, Cama y Fecha, pero no se coloca el Numero de afili- 


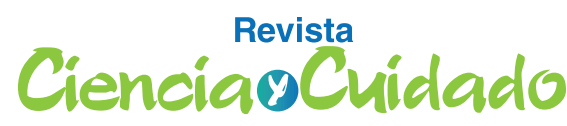

Scientific Journal of Nursing

ación, la Edad y el Sexo. Lo mismo pasa con el estándar sobre toma de los reactivos: destrostix/glucocetonurias cumpliendo solamente con la toma de destrostix. Acerca de lo que se dice de los dos reactivos anteriores Schachner B, et al., mencionaron en el IX Congresso Brasileiro de Informática em Saúde, en 2004, que cuando los formatos escritos de los servicios brindados no son completos y exactos, pueden ser utilizados como base legal para comprobar que la asistencia no se ha realizado según las normas aceptadas para su puesta en práctica, y por tanto pueden convertirse en prueba o evidencia para demandar la institución y al personal de la salud (33). Por otro lado, Cáceres et al. (8) refieren que a pesar de que se tiene plenamente identificada la importancia de los formatos de registros clínicos de enfermería, se han observado registros incompletos; es decir, que su incumplimiento parcial, puede ser por falta de formatos adecuados, por sobrecarga laboral o por desapego a los mismos; de donde sea importante contar con formatos de registros clínicos de enfermería completos que coadyuven a mejorar la continuidad del cuidado del paciente.

Hay que mencionar que se identificaron 23 estándares básicos referidos por la CONAMED, lo que supone que la hoja de registros clínicos de enfermería de la UCI si los cumple, representando un $57.5 \%$ de la totalidad de dicho formato. Por su parte García et al. (34), mencionan que la principal función de las notas de enfermería, a través de sus formatos consiste en priorizar el bienestar del paciente, por lo que son imprescindibles, específicas, para que contribuyan a la atención de la salud de las personas posibilitando el desarrollo de la disciplina. Desde otro punto de vista, López et al. (32) en su artículo de investigación titulado "Construcción y validación de un registro clínico para la atención asistencial de enfermería", realizado en México, especifican que los formatos para el registro de la atención de enfermería deben responder a las actualizaciones vigentes en materia normativa, de tal forma que permitan al personal de enfermería reflejar las intervenciones realizadas al paciente y reconocer el rol de los profesionales en la materia de salud, y con ello lograr mejorar la atención proporcionada en las UCIS a los pacientes.

Como contrapartida se localizaron 11 estándares con los que no cumple la hoja de registros clínicos de enfermería de la UCI de un hospital público en San Luis Potosí; de ahí que Aguilar y Martinez (15), señalan en su artículo de investigación titulado "La realidad
Evaluación de la hoja de enfermería de una Unidad

de Cuidados Intensivos de la Unidad de Cuidados Intensivos", realizado en 2017, que en México, los hospitales sólo ofrecen dos opciones de cuidado: la aplicable en las unidades de hospitalización convencional y la terapia intensiva, servicios de las unidades de salud en donde los pacientes requieren cuidado constante y atención especializada durante las 24 horas del día; además, el formato de la UCI de la institución en donde se realizó el estudio de investigación es ambiguo aunado a la infraestructura de la unidad, lo que hace que la hoja no logre cumplir en su totalidad con los estándares requeridos por la $\mathrm{CON}$ AMED.

Por último, se encontraron 4 estándares anexados por el personal de enfermería, a la hoja de registros clínicos de enfermería de la UCI de un hospital público en San Luis Potosí. Al respecto Carrillo, citado por Santana y Bauer (20) en su artículo de investigación "Calidad de los Cuidados de Enfermería y Satisfacción del paciente atendido en un Hospital de enseñanza" realizado en 2014, refiere que la documentación acerca de la práctica de enfermería, es necesaria para evaluar, mejorar la eficacia y la productividad. Sin embargo, los estándares agregados a la hoja por el personal de enfermería, como lo señala Villa (31), se tornan importantes debido a la complejidad de los cuidados proporcionados a través de la atención sanitaria, los límites de los profesionales de la salud y la capacidad de comunicación efectiva sobre la atención de los pacientes. Al respecto hay que agregar, que los formatos de registros clínicos de enfermería, son importantes dado que a través de ellos se puede evidenciar la implementación y cumplimiento de las acciones esenciales para la seguridad del paciente; igualmente permiten mejorar el actuar de la enfermería y su desempeño laboral, en pro de la salud de los pacientes.

\section{Conclusiones}

Se logró evaluar el nivel de cumplimiento de la hoja de registros clínicos de enfermería en la Unidad de Cuidados Intensivos de un hospital público de segundo nivel en San Luis Potosí; permitiendo identificar que existen algunos estándares que dan complimiento y otros que no. Al mismo tiempo se observa existen formatos de registro clínico de enfermería, que no cumplen con lo que establece la Comisión Nacional de Arbitraje Médico (CONAMED).

Con estos resultados se propuso y diseñó una nueva hoja de registros clínicos de enfermería para estas uni- 
dades de Cuidados Intensivos que contienen lo establecido por la Norma Oficial Mexicana 004-SSA3-2012, del Expediente Clínico; además de los lineamientos establecidos por los 40 estándares básicos de la Comisión Nacional de Arbitraje Médico (CONAMED), que coadyuve al personal de enfermería en su labor de registrar de manera integral el cuidado que se proporciona a los pacientes.

\section{Conflicto de interes}

Los autores declaran no tener ningun conflicto de intereses

\section{Referencias Bibliográficas}

1. Ponti L. La enfermería y su rol en la educación para la salud. [Internet], 10 de marzo de 2016. UAI Editorial [consultado 09 de agosto de 2021] Disponible en: https://www.teseopress.com/enfermeriayeducacion/ back-matter/conclusiones/

2. Young P, Hortis V, Chambi M, Finn B. Actualidad evangélica: Florence Nightingale (1820-1910), "madre" de la enfermería moderna [Internet]. Buenos Aires: Hospital Británico de Buenos Aires; 2014 [consultado 9 agosto de 2021]. Disponible en: https://www.actualidadevangelica.es/index.php?option=com content\&view=article\&id=7448:florence-nightingale-1820-1910-madre-de-la-enfermeriamoderna\&catid=43:destacados

3. Caumes G. La reglementation des écrits professionnels [The regulation of nursing record-keeping]. [en línea]. Mayo 2017. Rev Infirm. 66(231):18-19. [consultado 04 de junio de 2021]. Disponible en: https://www. j. revinf.2017.02.025. PMID: 28460722.

4. MEDwave. Importancia de los registros de enfermería. [en línea]. Biblioteca Digital de la Universidad Jorge Basadre. Mayo 2004. [consultado 04 de junio de 2021]. Disponible: https://www.medwave.cl/link.cgi/Medwave/Enfermeria/Mayo2004/2793

5. De Marinis M-G, Piredda M, Pascarella MC, Vincenzi B, Spiga F, Tartaglini D, Alvaro R, Matarese M. If it is not recorded, it has not been done. J Clin Nurse [Internet] 2010 junio [Consultado 09 de agosto de 2021]; 19 (11-12):1544-52. Disponible en: https://www.ncbi.nlm.nih.gov/pubmed/20438599

6. 6.-Machado DA, Figueiredo NMA. Registros de enfermería: el mensaje sobre la atención contenido en lenguaje escrito. Rev Research Care Fundam. 2010; 2 (Supl.): 293-7.

7. Méndez-Calixto G, Morales-Castañeda MA, Preciado-López ML, Ramírez-Núñez MR, Sosa- Gonzaga V, Jiménez y Villegas MC. La transcendencia que tienen los registros de enfermería en el expediente clínico. Revista Mexicana de Enfermería Cardiológica 2001; 9(1-4):11-17.

8. Cáceres-Castro J, Casihuman J, Paucar O. Factores relacionados a la calidad de los registros de enfermería (SOAPIE) en el servicio de emergencia del hospital militar central. [Internet] Lima, Perú, 2015. [consultado 4 de junio de 2021]. Disponible en: https://repositorio.upch.edu.pe/handle/20.500.12866/582

9. Nomura ATG, Silva MB, Almeida MA. Calidad de los registros de enfermería antes y después de la acreditación hospitalaria en un hospital Universitario. [Internet] Enfermería Rev Latino-Am. 2016; 24 : e2813. [consultado 9 de agosto de 2021]. Disponible en: http://dx.doi.org/10.1590/1518-8345.0686.2813

10. Diario Oficial de la Federación. NOM 168-SSA1-1998, DEL EXPEDIENTE CLINICO, [en línea], México, diciembre de 1998 [consultado 4 de junio del 2021] Disponible en: http://www.salud.gob.mx/unidades/cdi/ nom/168ssa18.html

11. Diario Oficial de la Federación. Norma Oficial Mexicana NOM-004-SSA3-2012, DEL EXPEDIENTE CLINÍCO. [en línea], México, 2012 [consultado 4 de junio de 2021] Disponible en: http://dof.gob.mx/nota detalle 
popup.php? codigo $=5272787$

12. Figueroa C, Mejía K, Santa Cruz K. Calidad de los registros electrónicos de enfermería y los factores que influyen en la Unidad de Cuidado Intensivo del Hospital Guillermo Kaelin de la Fuente [Tesis de Licenciatura en internet]. Perú: Universidad Peruana Cayetano Heredia; 2016 [consultado 09 de agosto de 2021]. 51p. Disponible en: http://repositorio.upch.edu.pe/handle/upch/595

13. Avilés-Reinoso L. Soto-Núñez C. Modelos de Enfermería en Unidades de Paciente Crítico: un paso hacia el cuidado avanzado. Enferm. glob. [en línea]. Murcia; abril 2014. 13(34). [consultado 04 de junio del 2021] Disponible en: http://scielo.isciii.es/scielo.php?script=sci arttext\&pid=S1695-61412014000200015

14. Hernández-Padilla JM. Ingreso, valoración, registros y alta del paciente crítico" refiere que el registro del seguimiento realizado al paciente críticos parte de las responsabilidades profesionales en una UCI. [en línea], diciembre 2015. [consultado 4 de junio de 2020]. Disponible en: https://www.researchgate.net/ publication/285536887 Ingreso_valoracion_registros_y alta del paciente critico/link/565ee61708aeafc2aac926dc/download

15. Aguilar-García CR, Martínez-Torres C. La realidad de la Unidad de Cuidados Intensivos. [Internet]. México; 2017. Med. Crit [Internet] 2017 [consultado 04 de junio del 2020]; 31(3):171-173. Disponible en: https:// www.medigraphic.com/pdfs/medcri/ti-2017/ti173k.pdf

16. Didry P. Regard sur l'évolution des écrits professionnels infirmiers [The evolution of nursing record-keeping]. Revue de l'infirmiere. 2017; 66(231), 20-21. https://doi.org/10.1016/j.revinf.2017.02.024

17. Collins S, Couture B, Kang MJ, Dykes P, Schnock K, Knaplund C, et al. Cuantificación y visualización de la carga de documentación de la hoja de flujo de enfermería en cuidados intensivos y agudos. AMIA ... [Internet]. Rev. Actas del Simposio Anual. Simposio AMIA. [Internet] 2018; 348-357. [consultado 04 de junio del 2021]. Disponible en: https://pubmed.ncbi.nlm.nih.gov/30815074/

18. Anderson EE. Issues surrounding record keeping in district nursing practice. British journal of community nursing, [in line]. 2000; 5(7), 352-356. [consulted June 4,2021]. Available in: https://doi.org/10.12968/ bjen.2000.5.7.7160

19. Comisión Nacional de Arbitraje Médico. Instructivo de requisición adecuada de la cédula para evaluar la eficacia de la práctica de Enfermería: 40 Estándares básicos. [Internet]. México; mayo 2017. [consultado 4 de junio de 2021]. Disponible en: http://www.conamed.gob.mx/gobmx/libros/pdf/instructivo.pdf

20. Santana J, Bauer A. Calidad de los Cuidados de Enfermería y Satisfacción del paciente atendido en un Hospital de enseñanza. Latino-Am Enfermagem. Junio; 2014; 22:454. [consultado 04 de junio del 2021] Disponible en: https://www.scielo.br/pdf/rlae/v22n3/es_0104-1169-rlae-22-03-00454.pdf

21. Diniz SOS, Silva PS, Figueiredo NMA, Tonini T. Calidad de registros de enfermería: reflexiones analíticas sobre sus formas y contenido. Rev Enferm UFPE. 2015; 9(10): 9616-23.

22. García-Ramírez S, Navío-Marco AM, Valentin L. Normas básicas para la elaboración de los registros de enfermería. [Internet], Madrid, España; 8-03-2007 Rev. Nure Investigación. [consultado 14 de abril del 2020]. Disponible en: https:/www.nureinvestigacion.es/OJS/index.php/nure/article/download/335/326

23. Hospital Regional Moquegua. Calidad de las notas de enfermería en la Unidad de Cuidados Intensivos. [Internet]. Perú; 2011. [consultado el 20 febrero de 2020]. Disponible en: http://tesis.unjbg.edu.pe:8080/handle/ unjbg/147

24. MEDwave. Importancia de los registros de enfermería. [Internet]. Biblioteca Digital de la Universidad Jorge Basadre. Mayo 2004. [consultado 20 febrero de 2020]. Disponible: https://www.medwave.cl/link.cgi/Medwave/Enfermeria/Mayo2004/2793

25. Quintana-Zavala M, Tinajero-González R. Investigación en enfermería en unidad de cuidado intensivo del adulto: una revisión de literatura. Horiz Enferm [Internet]. Enero 2019 [consultado 20 febrero de 2020]; 
Scientific Journal of Nursing

30(1):76-87. Disponible: http://publicaciones.horizonteenfermeria.uc.cl/index.php/rhe/article/view/259

26. Ventura-León J, Caycho-Rodríguez T, Barboza-Palomino M. ¿Cuántos participantes son necesarios? Un método para estimar el tamaño muestral en SEM. Arch Argent Pediatr. 2020;118: e510-3.

27. Asociación Médica. Declaración de Helsinki de la AMM - Principios éticos para las investigaciones médicas en seres humanos página principal en internet. Asociación Médica Mundial. [Internet], Actualizado mayo 2015, [consultado 23 de abril de 2020] Disponible en: https://www.wma.net/es/policies_post/declaración-de-helsinki-de-la-amm-principios-eticos-para-lasinvestigaciones-merdicas-en-seres-humanos/

28. Diario Oficial de la Federación. La Ley General de Salud en Materia de Investigación. [Internet], 07-021984. [Internet], Actualizado mayo 2015, [consultado 23 de abril de 2020] Disponible en: http://dof.gob.mx/ nota_detalle.php?codigo $=4652777 \&$ fecha $=07 / 02 / 1984$

29. Secretaría de Cultura. Código de conducta, etica y de prevención de conflictos de interés de la secretaría de cultura [Internet]. México: 2016 [citado 7 de mayo de 2020]. Disponible en: https://www.cultura.gob.mx/PDF/ Codigo-de-conducta-etica-y-Prevencion-de-Conflictos-de-Interes-de-la-Secretaria-de-Cultura-2016-08-24. pdf

30. Comisión Nacional de Arbitraje Médico. Código de Ética para Enfermeros. [Internet] [consultado 23 de abril de 2020] Disponible en: https://www.conamed.gob.mx/prof_salud/pdf/codigo enfermeras.pdf

31. Villa Suarez M. Registros de Enfermería como Evidencia del Cuidado. [Internet]. Diciembre 2013. $126-133$. Revista Ciencia e Innovación en Cuidado. [consultado 24 de agosto del 2021] Disponible en: http://revistas. unisimon.edu.co/index.php/innovacionsalud/article/download/59/45

32. López-Cocotle JJ, Moreno-Monsiváis MG, Saavedra-Vélez CH. Construcción y validación de un registro clínico para la atención asistencial de enfermería. Enfermeria Universitaria. [Internet]. Mexico; septiembre de 2017. [consultado 10 de junio del 2021] Disponible en: https://www.elsevier.es/es-revista-enfermeria-universitaria-400-pdf-S1665706317300611

33. Schachner B, Villalba E, Lores A, Baum A, Gómez A, López G, et ál. Informatización del proceso de Enfermería en un hospital de alta complejidad. IX Congresso Brasileiro de Informática em Saúde. 2004. Disponible en: http://www.hospitalitaliano.org.ar/archivos/servicios_attachs/2639.pdf

34. García M, Cárdenas L, Arana B, Monroy A, Hernández Y, Salvador C. Construcción emergente del concepto: cuidado profesional de enfermería. Texto Contexto Enferm, Florianópolis. 2011; 20: 74-80 